\title{
MS10-01 | Combatting MX Measurement Errors at Source
}

Evans, Gwyndaf (Diamond Light Source, Didcot, GBR)

Micro-crystal diffraction has, in the past two decades, played an essential role in the success of synchrotron macromolecular crystallography (SMX) allowing major progress to be made in structural biology and biomedicine [1]. It has however become clear that even with major advances in detectors, X-ray sources and optics, automation and software, micro-crystallography (crystal dimensions $<5$ microns) is fundamentally limited by crystal lifetime and, linked to this, the signal to noise ratio in diffraction data. Principal sources of noise come from X-ray background generated by disordered material in the sample (solvent regions in the crystal and solvent or other material surrounding the crystal) and scatter from air in the sample environment.

The VMXm beamline at Diamond Light Source has been developed to address these major sources of X-ray background and bring micro-crystallography closer to the true physical limits of microcrystal SMX. The presentation will describe novel approaches to X-ray focusing, crystal imaging, sample mounting and sample environments that together produce more than an order of magnitude reduction in X-ray background. The improved signal to noise ratio has the immediate effect of either improving the diffraction resolution limit from microcrystals, or extending the lifetime of crystals due to the fact that a reduced dose can deliver an equivalent signal-noise ratio. The results of benchmark comparison experiments will be presented together with early scientific results from VXMm.

[1] G. Evans, D. Axford, D. Waterman, R.L. Owen, Macromolecular microcrystallography, Crystallogr. Rev. 17 (2011) 105-142. 